\title{
Effects of acupuncture on behavioral, cardiovascular and hormonal responses in restraint-stressed Wistar rats
}

C.M. Guimarães ${ }^{1}$,

M.C.M. Pinge ${ }^{1}$, Y. Yamamura ${ }^{2}$ and L.E.A.M. Mello ${ }^{1}$

\author{
${ }^{1}$ Departamento de Fisiologia and \\ ${ }^{2}$ Departamento de Ortopedia e Traumatologia, \\ Universidade Federal de São Paulo, São Paulo, SP, Brasil
}

\author{
Correspondence \\ L.E.A.M. Mello \\ Departamento de Fisiologia \\ UNIFESP-EPM \\ Rua Botucatu, 862 \\ 04023-900 São Paulo, SP \\ Brasil \\ Fax: 55 (011) 571-5651 \\ E-mail: epmnf@eu.ansp.br \\ Research supported by $\mathrm{CNPq}$ and \\ PRONEX. C.M. Guimarães and \\ M.C.M. Pinge were the recipients \\ of $\mathrm{CNPq}$ fellowships. Publication \\ supported by FAPESP.
}

Received February 28, 1997 Accepted September 8, 1997

\begin{abstract}
Stress is a well-known entity and may be defined as a threat to the homeostasis of a being. In the present study, we evaluated the effects of acupuncture on the physiological responses induced by restraint stress. Acupuncture is an ancient therapeutic technique which is used in the treatment and prevention of diseases. Its proposed mechanisms EPM-1 rats were divided into four groups: group I $(\mathrm{N}=12)$, unrestrained rats with cannulas previously implanted into their femoral arteries for blood pressure and heart rate measurements; group II ( $\mathrm{N}=$ 12), rats that were also cannulated and were submitted to 60-min immobilization; group III $(\mathrm{N}=12)$, same as group II but with acupuncture needles implanted at points SP6, S36, REN17, P6 and DU20 during the immobilization period; group IV $(\mathrm{N}=14)$, same as group III but with needles implanted at points not related to acupuncture (nonacupoints). During the 60-min immobilization period animals were assessed for stress-related behaviors, heart rate, blood pressure and plasma corticosterone, noradrenaline and adrenaline levels. Group III animals showed a significant reduction $(60 \%$ on average, $\mathrm{P}<0.02)$ in restraint-induced behaviors when compared to groups II and IV. Data from cardiovascular and hormonal assessments indicated no differences between group III and group II and IV animals, but tended to be lower (50\% reduction on average) in group I animals. We hypothesize that acupuncture at points SP6, S36, REN17, P6 and DU20 has an anxiolytic effect on restraint-induced stress that is not due to a sedative action.
\end{abstract} Key words - Stress

- Acupuncture

- Catecholamines

- Corticosterone

\section{Introduction}

The concept of stress is very old; it must have occurred even to prehistoric man that the loss of vigor and feeling of exhaustion that overcame him after hard labor, prolonged exposure to cold or heat, loss of blood, agonizing fear, or any kind of disease had something in common (1).
The term stress has been used and occasionally abused by scientists and the general public in almost every language of the civilized world. Many definitions have been attributed to this word. Stress is synonymous to "strain" which itself has Indo-European roots. In ancient Greek it was the root of "strangale", and the verb "strangaleuin" meant to strangle. In Latin it was the root of 
the verb "stringere", meaning to hold, to press. Thus, the roots of the word stress always bear the meaning of adverse forces.

In a sense, stress may be defined as any threat to homeostasis. To achieve or maintain homeostasis adaptive reactions are developed by the body. Such reactions might be specific to a given agent or generic and nonspecific, depending more on the intensity of the stressor rather than on its nature (2).

Acupuncture is an ancient therapeutic technique which is used in the treatment and prevention of diseases. Its proposed mechanisms of action are based on the principle of maintaining or recovering homeostasis. Many efforts have been made to adequately establish the properties and characteristics of these mechanisms in terms of neuroanatomy, neurophysiology, and neurochemistry (3-13). However, most studies have been published in the Chinese language only and there are still many open questions. The use of acupuncture in clinical practice has gained wide acceptance over recent years but this has not been followed by a corresponding increase in basic or clinical research.

One of the many clinical uses of acupuncture is the treatment of stress. In the present study, we investigated the efficacy of clinically used acupoints (acupuncture points) on various physiological and behavioral parameters of rats submitted to restraint stress.

\section{Material and Methods}

Fifty adult male Wistar EPM-1 rats, provided by the Central Animal House of UNIFESP, weighing 250-400 g, were used in the present study. The animals were housed in groups of 5-6/cage, with free access to food and water in a vivarium with controlled light $(12 / 12 \mathrm{~h})$ and temperature $\left(23^{\circ} \mathrm{C}\right)$.

At least five days after arriving at our vivarium, animals were assigned to one of the following four groups: group I $(\mathrm{N}=12)$, rats that were unrestrained but had cannulas implanted into their femoral arteries for blood pressure and heart rate measurements; group II $(\mathrm{N}=12)$, rats that were also cannulated and were submitted to 60-min immobilization; group III ( $\mathrm{N}=12)$, same as group II but with acupuncture needles implanted at points SP6, S36, REN17, P6 and DU20 during the 60-min immobilization period; group IV ( $\mathrm{N}$ =14), same as group III but with needles implanted at points not related to acupuncture (non-acupoints).

One day prior to the experiment each animal was implanted with a catheter (PE50) into the femoral artery under sodium thiopental anesthesia $(40 \mathrm{mg} / \mathrm{kg}$, ip) with the help of a surgical microscope. At the same time, the skin was submitted to tricotomy in a few selected areas and acupoints were located by the measurement of skin conductance with a Plexus T20 (Lautz, São Paulo, SP, Brazil) equipment. Initial selection of these skin areas was based on available acupoint charts for rodents and anatomical references such as bones and joints (3). The skin over the acupoints determined in this way was marked with a felt pen (for needle insertion on the next day). The acupoints and neighboring non-acupoints were further characterized by measuring the skin conductance for a 5-V pulse applied $10 \mathrm{~mm}$ from an acupoint (or non-acupoint). A non-acupoint corresponding to a given acupoint was chosen on the basis of its signal being less than half of the conductance measured for that acupoint (7). After this procedure animals were housed overnight in individual cages. Acupoints were chosen according to Lin et al. (5) and Liao et al. (6).

On the following day, starting between 9:00 and 11:00 a.m., group I animals housed in the same cages as those used after cannulation were evaluated for heart rate and blood pressure for $60 \mathrm{~min}$. Mean blood pressure was measured with a P23 Db pressure transducer connected to a Beckman R511A polygraph. Heart rate was measured with a 
Beckman 9875B cardiotachometer.

Also on the day after cannulation, starting between 9:00 and 11:00 a.m., group II, III and IV animals were submitted to a 60min episode of restraint stress on a wooden board and had their heart rate and blood pressure measured as described above. Restraint was performed with the animal placed on its back, its forelimbs perpendicularly stretched in relation to the rat's main body axis and its hind limbs maintained in quasiflexion at approximately 45 degrees in relation to the same axis. Limbs and body were secured in place with the aid of adhesive tape, which was fastened to the wooden board. Immediately after being restrained group III animals had acupuncture needles implanted at points SP6, S36, REN17, P6 and DU20 throughout the immobilization period. Similarly, group IV animals had needles implanted at points not related to acupuncture (non-acupoints) throughout the immobilization period. Needles were commercially available Chinese acupuncture needles (No. 82, size 0.5 ) with a diameter of $0.3 \mathrm{~mm}$ and a length of $2 \times 1.3 \mathrm{~cm}$ (handleneedle).

In addition, these animals had their restraint-induced behaviors recorded. Usual behaviors included intense or mild head movements, body jerks, vocalization, masticatory movements, and feces and urine elimination. Such behaviors were measured as frequency of occurrence for $60 \mathrm{~min}$ regardless of whether or not they also carried a time component. Flexion of the head immediately followed by relaxation (a return to the former position) characterized "head movements". Flexions of the head that were not immediately followed by relaxation (usually maintained over 4-8 s) characterized "maintained head movements". As for head movements, "mild body jerks" were phasic/instantaneous events as compared to longer lasting/tonic (usually maintained over 4-8 s) "intense body jerks". Measurement of the above restraintinduced behaviors was based on a review by
Archer (14). Restraint-induced behaviors were not evaluated for group I animals since they were not restrained.

After the 60-min period each animal (groups I-IV) was decapitated and blood was collected for the determination of plasma corticosterone, noradrenaline and adrenaline levels. Due to technical problems the blood of some animals was not processed for all hormones or the data was discarded. The number of animals with valid results for each hormone in groups I, II, III and IV, respectively, was $11,7,8$ and 7 for corticosterone, 8, 6, 5 and 6 for noradrenaline, and 7, 7,6 and 6 for adrenaline. Plasma corticosterone concentration was assessed by the Guillemin fluorimetric method (15). Noradrenaline and adrenaline were measured by an HPLC method (16). Corticosterone levels are reported as mean $\pm \mathrm{SD}$, and noradrenaline and adrenaline levels are reported as mean (range) due to the high variability of the results (which therefore could not be expressed as mean \pm SD or SEM). Differences were considered to be statistically significant when $\mathrm{P} \leq 0.05$ (Kruskall-Wallis).

\section{Results}

The results for the behaviors executed during the $60 \mathrm{~min}$ of restraint stress are summarized in Table 1. Maintained head

Table 1 - Mean number of behaviors during 60 min of restraint stress in animals previously submitted to the application of acupuncture needles at acupoints (group III), or at non-acupoints (group IV), or not submitted to needle application (group II).

aP $<0.01$ (group III x group II or IV); bP $<0.02$ (group III $x$ group IV); ${ }^{c P}<0.005$ (group III $x$ group II or IV); $\mathrm{d}$ < $<0.01$ (group III $x$ group II) (Kruskall-Wallis).

\begin{tabular}{lccc}
\hline & $\begin{array}{c}\text { Group II } \\
(\mathrm{N}=12)\end{array}$ & $\begin{array}{c}\text { Group III } \\
(\mathrm{N}=12)\end{array}$ & $\begin{array}{r}\text { Group IV } \\
(\mathrm{N}=14)\end{array}$ \\
\hline Head movements & 24.4 & 17.2 & 33.7 \\
Maintained head movements & 16.2 & $4.2^{\mathrm{a}}$ & 21.1 \\
Mild body jerk & 8.1 & $3.2^{\mathrm{b}}$ & 12.0 \\
Intense body jerk & 30.7 & $10.8^{\mathrm{c}}$ & 32.8 \\
Masticatory movements & 5.6 & 2.2 & 2.4 \\
Feces bolus & 4.2 & $1.5^{\mathrm{d}}$ & 2.6 \\
Vocalizations & 7.2 & 3.1 & 11.0
\end{tabular}


movements were long-lasting behaviors of more than a few seconds, in contrast to head movements which were just simple (almost reflex) movements. Similarly, intense body jerks represented relatively long-lasting and vigorous attempts to move the body (escape?) as compared to mild body jerks which were short and weak attempts.

There were no significant differences in heart rate or blood pressure between the 4 groups. In general, the blood pressure, but not the heart rate, of group I tended to be lower than that of the other groups. No tendency was seen over time $(60 \mathrm{~min})$ for blood pressure to accommodate to lower levels for any of the groups. However, a marked (but not significant) tendency for an increase in heart rate over time was observed for group III animals when compared to the other groups.

Plasma corticosterone levels were significantly lower $(\mathrm{P}=0.006$, Kruskal-Wallis test $)$ for group I $(23.2 \pm 9.1 \mu \mathrm{g} / \mathrm{dl})$ as compared to groups III and IV $(37.4 \pm 8.9$ and $37.5 \pm 7.7$ $\mu \mathrm{g} / \mathrm{dl})$. Similarly, plasma noradrenaline and adrenaline levels tended to be lower in group I as compared to the other groups (on average group I values were $45 \%$ of the values of the other groups), but were significantly lower

Table 2 - Mean plasma levels of corticosterone, noradrenaline and adrenaline in unrestrained animals (group I) and immediately after $60 \mathrm{~min}$ of restraint stress in animals previously submitted to the application of acupuncture needles at acupoints (group III), or at non-acupoints (group IV) or not submitted to needle application (group II).

Corticosterone levels are reported as mean $\pm \mathrm{SD}$ in $\mu \mathrm{g} / \mathrm{dl}$; noradrenaline and adrenaline levels are reported as mean (range) in $\mathrm{pg} / \mathrm{ml}$. Corticosterone: Kruskal-Wallis, $\mathrm{P}<0.005$; multiple comparison, a $\mathrm{P}<0.01$ (group I<group III or IV); noradrenaline: Kruskal-Wallis, $\mathrm{P}<0.03$; multiple comparison, $\mathrm{b} P<0.02$ (group $\mathrm{I}<$ group IV); adrenaline: Kruskal-Wallis, $\mathrm{P}<0.02$; multiple comparison, ${ }^{\mathrm{C}} \mathrm{P}<0.05$ (group $\mathrm{I}<$ group IV).

\begin{tabular}{lcccc}
\hline & Group I & Group II & Group III & Group IV \\
\hline Corticosterone & $23.2 \pm 9.1$ & $35.7 \pm 9.2$ & $37.4 \pm 8.9^{a}$ & $37.5 \pm 7.7^{a}$ \\
& $(\mathrm{~N}=11)$ & $(\mathrm{N}=7)$ & $(\mathrm{N}=8)$ & $(\mathrm{N}=7)$ \\
Noradrenaline & $175(305-95)$ & $403(971-191)$ & $274(420-95)$ & $485(1131-226)$ \\
& $(\mathrm{N}=8)$ & $(\mathrm{N}=6)$ & $(\mathrm{N}=5)$ & $(\mathrm{N}=6)$ \\
Adrenaline & $320(632-129)$ & $643(1474-232)$ & $509(826-180)$ & $953(1810-548)$ \\
& $(\mathrm{N}=7)$ & $(\mathrm{N}=7)$ & $(\mathrm{N}=6)$ & $(\mathrm{N}=6)$
\end{tabular}

only when compared to group IV $(\mathrm{P}=0.024$ for noradrenaline and $\mathrm{P}=0.02$ for adrenaline, Kruskal-Wallis test). For a detailed view of corticosterone, noradrenaline and adrenaline levels refer to Table 2.

\section{Discussion}

Our results indicate that stimulation of acupoints SP6, S36, REN17, P6 and DU20 significantly attenuated restraint-induced behaviors. The finding that restraint stress did not increase blood pressure or heart rate is in agreement with previous reports (17). The results of the 2 control groups for stressassociated behaviors (groups II and IV) were relatively similar and on average almost 3 times more frequent than those of group III. Taken together, these results suggest that stimulation of the above acupoints reduced stress-associated behaviors through a mechanism that is not merely related to needle insertion but specifically to needle insertion into acupoints.

The behaviors measured to assess the effects of acupuncture on restraint stress have been identified as restraint-induced behaviors (14). Behavioral responses to stress may be classified as either coping or defense (18). In fact, such distinction may be hard to establish and many investigators try not to use it (19), since defense may imply fight/ flight as well as freezing (18). In terms of the behavioral responses observed for group III animals, it is extremely difficult for us to classify such attenuation of the behavioral response as either defense or coping strategy. Even though coping is usually considered to be a learning strategy, it is possible that the animals did learn such strategy during the 60-min trial.

It is possible that group III animals were quieter than groups II and IV due to pain inflicted by the needle. Accordingly, by having their movements restricted group III animals would suffer less pain. This hypothesis however, is unlikely because it does not 
apply to group IV animals, which also had implanted needles. On the other hand, the existence of anatomical differences between acupoints and non-acupoints (3) does not rule out the above hypothesis. However, consideration of the other physiological measurements (blood pressure, heart rate, hormonal levels) also does not help to support the pain hypothesis since stress + pain should provoke higher blood pressure, heart rate and hormonal levels.

Another hypothesis to explain our findings is that of sedation. Group III animals could be sedated and thus display less frequent restraint-induced behaviors. Here again, the other physiological measurements would argue against such a hypothesis since none was decreased in group III animals. In humans, however, such acupoints are classically associated with relaxation and sedation $(13,20)$. A compromise hypothesis would have to consider that stimulation of the acupoints in this case would cause a dissociation between behavioral (cognitive, emotional) and body responses (heart rate, blood pressure and hormonal levels).

However, a much simpler explanation for the apparent dissociation between diminished behavior and the unchanged additional physiological measurements is the time-scale for each one. In the cascade of events triggered by a stressing agent, restraint in our case, within milliseconds there is the activation of brain structures that lead to pituitary and adrenal activation within seconds, cortisol activation by tenths of minutes, and immunoglobulin activation within days and weeks (18). Thus, behavioral manifestations could be a much more sensitive index, with better temporal resolution and with little or no contamination by previous stresses as compared to the hormones measured in this experiment.

In contrast to our findings, Liao and colleagues (6) reported that electroacupuncture of point S36 (also used in our experiment) reduced corticosterone levels in a model of restraint stress in rabbits. Among the many differences between their protocol and ours (rabbit $\mathrm{x}$ rat; 2 x 8 acupoints; electric $\mathrm{x}$ manual acupuncture), one which might have been critical is the time chosen for blood collection (6:00 p.m. x 10:00 a.m.). The moment chosen for blood collection in their experiment (6:00 p.m.) corresponds to the daily peak of corticosterone secretion (21). It has recently been demonstrated that even a single exposure to stressing agents may increase morning corticosterone peaks while not affecting circadian rhythm or afternoon peaks (22).

The general understanding of the stress response involves an orchestrated action of many levels of the organism including the brain, pituitary, adrenal and many body systems. The putative anxiolytic effects of acupuncture suggested by the present results indicate that acupuncture may have an effect on the stress response. At present, we do not know which mechanisms are involved in such action. Further research is needed to assess how a peripherally applied, and seemingly unrelated, stimulation may exert effects on centrally driven processes. 


\section{References}

1. Selye $H$ (1973). The evolution of the stress concept. American Scientist, 61: 692-699.

2. Chrousos GP, Loriaux DL \& Gold PW (1988). Mechanisms of physical and emotional stress. Advances in Experimental Medicine and Biology, 245: 3-11.

3. Tirgoviste Cl (1975). Anatomic and functional particularities of skin areas used in acupuncture. American Journal of Acupuncture, 3: 199-206.

4. Wei LY \& Hodson C (1977). Nerve transmission and acupuncture mechanisms. American Journal of Acupuncture, 5: 6983.

5. Lin MT, Liv GG, Soong JJ, Chern YF \& Wu KM (1979). Effects of stimulation of acupuncture loci Ta-Chuei (GO-14), Nei-Kuan (EH-6) and Tsu san-Li (St-36) on thermoregulatory function of normal adults. American Journal of Chinese Medicine, 7: 324-332.

6. Liao YY, Seto K, Saito H, Fujita M \& Kawakami M (1980). Effects of acupuncture on adrenocortical hormone production: II. Effect of acupuncture on the response of adrenocortical hormone production to stress. American Journal of Chinese Medicine, 8: 160-166.

7. Zong-xiang Z (1981). Research advances in the electrical specificity of meridians and acupuncture points. American Journal of Acupuncture, 9: 203-216.

8. Sekhan MS \& Khatri HL (1982). Acupuncture channels - myth or reality? Chinese Medical Journal, 95: 912-914.
9. Wong WH \& Brayton D (1982). The physiology of acupuncture: Effects of acupuncture on peripheral circulation. American Journal of Acupuncture, 10: 59-63.

10. Wu DZ (1990). Acupuncture and neurophysiology. Clinical Neurology and Neurosurgery, 92: 13-25.

11. Bing Z, Villanueva L \& Bars DL (1991). Acupuncture evoked responses of subnucleus reticularis dorsalis neurons in the rat medulla. Neuroscience, 44: 693-703.

12. Anderson $S$ (1993). The functional background in acupuncture effects. Scandinavian Journal of Rehabilitation Medicine, 29 (Suppl): 31-60.

13. Yamamura Y (1993). Acupuntura-Tradicional: a Arte de Inserir. Roca, São Paulo.

14. Archer J (1973). Tests of emotionality in rats and mice: a review. Animal Behavior 21: 205-235.

15. Guillemin R, Clayton GW, Lipscomb HS \& Smith JD (1959). Fluorimetric measurement of rat plasma and adrenal corticosterone concentration. Journal of Laboratory and Clinical Medicine, 53: 830-832.

16. Mazzacoratti MGN, Casarine DE, Fernandes MJS \& Cavalheiro EA (1992). Serum catecholamine levels determined by high performance liquid chromatography coupled with electrochemical detection. Arquivos Brasileiros de Endocrinologia e Metabologia, 36: 119-122.
17. Paula RB, Marson O, Tufik S, Kohlmann Jr O, Bossolan D, Ginosa M, Neves FAR \& Ramos OL (1990). Stress and hypertension: studies in three experimental models. Revista do Hospital São Paulo, 2: 1922.

18. Ursin H \& Olff M (1993). The stress re sponse. In: Stanford SC \& Salmon P (Editors), Stress: From Synapse to Syndrome. Academic Press, New York.

19. Lazarus RS \& Folkman S (1984). Stress Appraisal and Coping. Springer-Verlag, New York.

20. Nghi NV (1981). Patogenia y Patologia Energeticas en Medicina China. Tratamento por Acupuntura y Masajes. Instituto de Acupuntura Médica de Madrid, Madrid.

21. Retiene K, Zimmerman E, Schindler WJ, Neuenschwander J \& Lipscomb HS (1968). A correlative study of endocrine rhythms in rats. Acta Endocrinologica, 57: 615-622.

22. Ottenweller JE, Servatius RJ \& Natelson BH (1994). Repeated stress persistently elevates morning but not evening plasma corticosterone levels in male rats. Physiology and Behavior, 55: 337-340. 